\title{
Mentoria no curso de Medicina: desafios da metodologia ativa de aprendizagem durante a pandemia de Covid-19
}

\section{Mentoring at medical school: Challenges of active learning methodology during the COVID-19 pandemic}

\author{
Vera Lucia Nascimento Blaia d'Avila' 이 | verablaia@gmail.com \\ Wilson Luvizotto Medina' (1) wlmedina@terra.com.br \\ Nicoli Abrão Fasanella' (1) nicoli.abrao@gmail.com \\ Paulo Henrique Pires de Aguiar' 1 (1) \\ Godofredo Campos Borges' (1) gcborges@pucsp.br \\ Sandro Blasi Esposito' 10 sbesposito@pucsp.br
}

\section{RESUMO}

Introdução: A atividade de mentoria pode ser vista como um relacionamento especial entre mentor e mentorado que pode contribuir para a transição entre o ensino médio e a universidade. No ano de 2020, com a pandemia de Covid-19, houve a necessidade de migração súbita das atividades presenciais acadêmicas para o ensino utilizando as plataformas digitais, o que desencadeou mudanças na estratégia pedagógica das escolas médicas e, consequentemente, impactou as atividades de mentoria.

Relato de experiência: Por meio de análise do discurso dos mentores do primeiro ano do curso de Medicina de uma universidade particular, ao longo do ano de 2020, discutimos os registros que ocorreram em tempo real após cada encontro semanal em sala virtual exclusiva, na qual os mentores podiam compartilhar livremente suas impressões, considerações e depoimentos sobre o desenvolvimento de cada sessão de mentoria.

Discussão: A mentoria não trata apenas do desempenho acadêmico, mas também proporciona uma visão mais ampla das questões relacionadas ao estudante e ao seu contexto, favorecendo o desenvolvimento do docente, do discente e da instituição. A mudança para as plataformas digitais não se mostrou um problema, com rápida adaptação dos envolvidos. A percepção de pertencimento e a participação do grupo foram fatores importantes no desenvolvimento da mentoria, e o acolhimento saudável dos estudantes e mentores demonstrou um fortalecimento no senso de auto humanidade.

Conclusão: Estudantes e mentores cultivaram um espaço para discussão de suas rotinas no desenvolvimento do ensino e aprendizagem. Diante do cenário epidemiológico atípico, houve a percepção de que os estudantes enfrentaram problemas semelhantes e buscaram, em conjunto, sugestões para soluções. Os mentores concluíram que uma programação comum, formal, para as sessões de mentoria não deveria ser aplicada rotineiramente.

Palavras-chave: Educação Médica; Tutoria; Desempenho Acadêmico; Covid-19.

\section{ABSTRACT}

Introduction: The mentoring activity can be seen as a special relationship between mentor and mentee, which can contribute to the transition from high school to university. In 2020, amid the Covid-19 pandemic, there was a sudden shift from face-to-face academic activities to meetings using digital platforms, triggering changes in the educational strategy of medical schools, and, consequently, impacting mentoring activities.

Experience report: Through discourse analysis of accounts given by mentors of first year medical students at a private university in 2020, we discussed the records made in real time after each weekly meeting in an exclusive virtual room, in which they could freely share their experiences, impressions, considerations and testimonies about the development of each mentoring session.

Discussion: Mentoring is not just about academic performance and provides a broader view of issues related to the student and their context, favoring the development of the teacher (mentor), student (mentee) and even the institution. The change to digital platforms was not a problem, as those involved swiftly adapted accordingly. The perception of belonging and participation in the group were important factors in the development of mentoring and the healthy reception of students and mentors demonstrated a strengthening in the sense of self humanity.

Conclusion: Mentees and mentors cultivated a space for discussing their routines in the development of teaching and learning. In view of the atypical epidemiological scenario, there was a perception that students faced similar problems and sought suggestions for solutions together. Mentors concluded that a common, formal schedule for mentoring sessions should not be applied routinely.

Keywords: Medical Education; Mentoring; Academic Performance; Covid-19.

${ }^{1}$ Pontifícia Universidade Católica de São Paulo, Sorocaba, São Paulo, Brasil.

Editora: Lia Marcia Cruz da Silveira

Recebido em 03/03/21; Aceito em 22/03/21.

Avaliado pelo processo de double blind review. 


\section{INTRODUÇÃO}

A atividade de mentoria pode ser vista como um relacionamento especial que se desenvolve como uma via de mão dupla, mas no início do curso de Medicina pode ser de mão única, com o mentor dirigindo a conversa e as atividades ${ }^{1,2}$. À medida que o relacionamento amadurece, a orientação aos estudantes pode estar associada a uma maior reflexão do mentor, impactando sua maneira de conduzir as discussões e ponderações ${ }^{1,3}$. O contato frequente associado à energia e ao entusiasmo dos estudantes pode provocar novas formas de desenvolvimento individual e proporcionar, aos estudantes e ao mentor, maior satisfação acadêmica com consolidação gradativa da parceria na busca do aprender a aprender ${ }^{3}$.

A transição entre o ensino médio e a educação superior foi identificada como um período muito estressante para os estudantes ${ }^{4}$. As intervenções realizadas nessa fase de mudança, com a oportunidade de convívio constante na mentoria, podem ser muito relevantes no auxílio aos estudantes nesse período de desafios e se tornam um meio pelo qual se pode promover o desenvolvimento profissional e pessoal. Essas mediações podem também ter impacto positivo no período de transição e manter o bem-estar especialmente nos estudantes do primeiro ano da escola médica 5 .

No surreal ano de 2020, com a pandemia da coronavirus disease 2019 (Covid-19) que provocou uma mudança ímpar na estratégia do ensino médico aos estudantes de graduação, gestores, docentes e discentes foram obrigados a se esforçar, a reaprender ou adquirir novas habilidades para o desenvolvimento de atividades exclusivamente on-line ${ }^{6}$.

Nesse contexto, todas as atividades presenciais características do currículo médico voltado para metodologias ativas de aprendizagem de nossa escola ${ }^{7,8}$, com inserção dos estudantes desde a primeira série em cenários de prática, foram substituídas por cenários virtuais, associadas ao desenvolvimento de tutorias, sustentações teóricas e práticas exclusivamente a distância durante sete meses, com retorno de atividades práticas presenciais de modo seguro e em pequenos grupos no último trimestre do ano acadêmico.

Diante da realidade imposta e da premissa de que as atividades remotas poderão seguir no ano acadêmico de 2021, fez-se necessário o compartilhamento de experiências para entender o impacto da atividade de mentoria na inserção do estudante de primeira série do curso de Medicina no contexto acadêmico durante a pandemia de Covid-19 e se a mentoria pode ser considerada ferramenta significante no gerenciamento do aprendizado e no desempenho acadêmico dos estudantes da primeira série do curso de graduação, sob a perspectiva das impressões dos mentores.

\section{RELATO DE EXPERIÊNCIA}

Trata-se de um relato de experiência baseado na análise do discurso dos registros, das impressões e dos depoimentos feitos pelos mentores do primeiro ano do curso de Medicina ao longo do ano de $2020^{\circ}$. As interações habituais entre os estudantes das diversas séries e as interações estudante/docente sofreram interrupção abrupta e obrigatória no início do ano acadêmico, após o decreto do Ministério da Educação com a suspensão das atividades presenciais em todos os níveis de ensino por meio da Portaria no 345, de 19 de março de $2020^{10}$. No contexto do curso de Medicina da nossa escola, as atividades dos estudantes ingressantes foram desenvolvidas de forma presencial por escassos 20 dias e, após um hiato de 15 dias, a coordenação de curso redirecionou as atividades acadêmicas de todas as séries para uma plataforma virtual institucional exclusiva para desenvolvimento das atividades curriculares.

Ao início do ano, foram inseridos 11 docentes como mentores em Estudos Orientados, denominação formal institucional da atividade de mentoria, com periodicidade semanal e duas horas de duração, para acompanhar os 110 estudantes ingressantes no curso médico da instituição. Todos os docentes já participavam de atividades acadêmicas regulares com os estudantes de graduação, e a grande maioria atuava há mais de dez anos em subespecialidades médicas, clínicas ou cirúrgicas, agregando à sua prática acadêmica as atividades de mentoria. Três docentes já haviam participado de atividades de mentoria em 2019, e oito estavam debutando. O compromisso docente foi pactuado com o objetivo de seguimento de seus grupos de mentoria ao longo dos três primeiros anos do curso.

Essa análise foi discutida reservadamente com todos os mentores e realizada a partir do resgate das postagens escritas e armazenadas em uma sala virtual privativa. Os depoimentos foram compartilhados em tempo real, após cada encontro semanal de mentoria em 2020. Para expor as dificuldades acadêmicas enfrentadas nesse ano atípico de migração de atividades presenciais para atividades a distância, cumprindo rigorosamente o conteúdo proposto no projeto pedagógico, os resultados foram apresentados em ordem cronológica de acontecimentos.

Ao início do ano acadêmico, todos os docentes do grupo participaram de capacitação para a atividade. Na primeira reunião presencial de cada mentor com seu grupo de dez estudantes, foram discutidas as ações iniciais, baseadas em autoconhecimento do grupo, na assinatura de um "contrato de confidencialidade" que pactuava a segurança das informações compartilhadas naquele ambiente e algumas regras para o bom desenvolvimento da atividade, como tempo de tolerância de atrasos, assiduidade e formação de um grupo privado em 
rede social. Esses espaços visavam a uma interação e parceria acadêmica, permitindo trocas de experiências, angústias, dúvidas de diversas etiologias sem o compromisso formal do aprendizado e avaliação. Nas mensagens iniciais entre os mentores na rede social, observamos um consenso da necessidade de uma breve reunião entre os mentores, de 30 minutos, antes de cada sessão de mentoria, para estabelecer os principais pontos que seriam comuns para discussão em cada semana, contemplando a demanda originada da atividade acadêmica semanal ou algum evento adicional.

Após duas semanas de aulas, a universidade suspendeu todas as aulas presenciais por determinação da Secretaria de Saúde, dando início ao processo de implantação de um ambiente virtual de atividades acadêmicas e capacitação docente em plataforma institucional. Pelo ineditismo da atividade para muitos docentes, tanto as reuniões de capacitação em ambiente virtual institucional como o diálogo na rede social privativa, entre os mentores, foram fundamentais para manter o otimismo e as recomendações de necessidade de preservar ambientes seguros para os estudantes e seus familiares.

Em abril 2020, reiniciamos as atividades de mentoria na plataforma institucional e, em todos os grupos, os registros apontavam que os estudantes compartilharam suas inseguranças sobre o cenário nacional, pontuando as dificuldades. Foi pactuado com a coordenação do curso que as reuniões seriam gravadas somente para registro da presença, mas as discussões seriam feitas em ambiente protegido, sem gravação.

Quanto à programação das atividades das mentorias, durante esse mês de adaptação, foram abordados vários temas, sendo os principais relacionados com a pandemia de Covid-19, as preocupações com os avós e pais e o impacto do retorno de muitos estudantes às suas casas de origem. Muitos estudantes voltaram para suas casas depois de algum tempo fora e essa adaptação foi gradativa, alguns com facilidade e outros sentindo falta da autonomia que adquiriram. Ainda assim, demonstravam otimismo quanto ao desafio das atividades acadêmicas em plataforma on-line, de fácil adaptação para essa geração e com o "bônus" de estudar revisando as aulas gravadas. Com a participação da coordenação de eixo do ano em sintonia com os mentores, foi possível acompanhar nessas mensagens o reflexo da realidade dos estudantes para além da mentoria.

Ao final dos dois primeiros meses de atividades, os registros dos mentores referiam que os estudantes estavam apresentando cansaço significativo, sentiam que recebiam mais tarefas do que seria o habitual, ficavam em frente das telas por períodos muito longos, dando sensação de sobrecarga de conteúdo em todas as "disciplinas". Mescladas às discussões do desenvolvimento acadêmico, as sessões de mentoria permitiam reflexões mais ecléticas, e temas relacionados ao lazer e "fique em casa" foram frequentemente estimulados, com discussões sobre séries, filmes e livros recomendados pelos mentores e pelos estudantes, permitindo sempre uma conversa amiga e confortável, e "o tempo passava tão rápido que nem percebíamos".

Nesse primeiro bimestre, discutimos, em várias ocasiões, as atividades relacionadas ao uso da plataforma institucional, com sugestões, críticas e fortalezas; o papel do médico e da equipe de saúde naquele momento, como consultores e atores no atendimento, sempre evitando exageros em uma situação atípica; a rotina diária de cada um; e a adaptação à nova realidade de vida e de estudo. A maioria já estava com rotina de estudo estabelecida, comentava que as atividades estavam muito boas e sentia-se amparada pelos diversos docentes nas atividades remotas. Os docentes já referiam maior adaptação, como pontuou um dos mentores:

Acredito que o processo da mentoria tem sido muito positivo. Um fórum onde discutimos ansiedades no enfrentamento das incertezas que vivemos no momento em vários cenários! Meu grupo é bem positivo! Vejo com bons olhos a evolução dos estudantes!

Alguns temas descritos foram desencadeados por algum evento, como discussões sobre abuso de drogas e álcool, causas, consequências, aspectos evolutivos e padrão de uso na população, nos estudantes. Outro assunto que surgiu diante do cenário político e social que vivemos foi o poder de algumas figuras históricas no controle das pessoas, levando a discussões sobre os tiranos do século passado, como Stalin, Hitler e Mao Tsé-tung, e as observações dos estudantes transbordavam opinião e conhecimento sobre história. A discussão sobre os motivos que os levaram a escolher a Faculdade de Medicina surgiu em todos os grupos. Todos participavam e faziam intervenções quando julgavam oportunas.

Com a aproximação do período de avaliação somativa, o foco das discussões mudou e os mentores referiam que os estudantes passaram a refletir sobre a importância dos temas das tutorias e sustentações na formação médica, dificuldades na condução dos estudos e aparentes diferenças de conhecimento entre eles; além disso, participavam espontaneamente na demanda de auxílio interpares aos discentes que ingressaram no curso mais tardiamente.

Em meio à ansiedade pelas avaliações, foi possível observar uma notável evolução referida nas discussões sobre as atividades de tutoria, habilidades e práticas em atenção à saúde, inclusive com os estudantes que ainda não conheciam a cidade, pelo ingresso após o início das atividades remotas. Nesse período, discutiram-se os formatos das avaliações, a 
importância da construção adequada de mapas conceituais e seu objetivo no contexto do estudo/aprendizagem.

Em várias ocasiões, os mentores de todos os grupos mencionaram que os estudantes externavam a preocupação com familiares e amigos internados, com registros de algumas perdas, alguns passando pelo processo de luto ou adoecimento decorrente da Covid-19 e relatavam maior ansiedade e dificuldade em lidar com o momento. Nesse contexto, discussões sobre a eutanásia, distanásia e ortotanásia, os conceitos de pandemia e a importância do Sistema Único de Saúde (SUS) mesclavam-se às orientações sobre o estudo, as formas de lidar com a ansiedade e a necessidade de manter foco e calma acima de tudo. Todos os mentores apontaram que os estudantes referiam muitas horas de estudo, sem tempo para outras atividades, e que ainda assim sentiam-se inseguros para as avaliações. Para vários discentes, os fatores externos significativos atrapalhavam a concentração no estudo, o que levou a reflexões importantes, com discussões sobre a forma de administrar as muitas distrações em casa, a necessidade de compartilhamento do computador e as tarefas demandadas pelo isolamento. De acordo com os estudantes, a mentoria ajudava na manutenção da saúde mental e proporcionava equilíbrio com o compartilhamento das dificuldades.

No final do primeiro semestre e início do segundo, mais confiantes na importância das intervenções semanais, os mentores avaliaramo processo da mentoria como muito positivo, como um fórum de discussão de ansiedades no enfrentamento das incertezas no momento dos acontecimentos, em vários cenários, e sentiram a evolução dos estudantes. Observaram e referiram também poucas alusões à pandemia durante os períodos pré e pós-avaliações somativas.

Uma situação interessante registrada foi a elaboração de quase um mapa conceitual das avaliações, no qual os discentes refletiram sobre a avaliação formativa das tutorias e a importância do feedback para orientação ao estudo. Não se queixaram de fazer avaliações on-line, parecendo quase natural para eles. Entretanto, as discussões sobre a qualidade das questões referentes aos objetivos de aprendizagem em cada módulo e a rigidez nas correções foram temas exaustivamente colocados nos registros de várias sessões de mentoria ao longo do ano. As avaliações que exigiam conhecimentos de habilidades e das aulas práticas remotas, em especial de anatomia, foram complexas e geraram muitas angústias, corrigidas após o retorno presencial.

A interação do grupo docente da mentoria com a coordenação de série foi um fator que contribuiu positivamente para as devolutivas das avaliações somativas. A análise dos registros demonstrou que a mentoria assumiu várias vezes, principalmente no segundo semestre, um papel importante em relação aos estudantes nas sugestões de planos de ações e metas para recuperação das pendências que surgiam no decorrer do estudo dos módulos, lembrando-os da espiral construtivista ${ }^{11}$. Havia um espaço reservado dentro do horário das sessões para reflexões voltadas ao fortalecimento da autoestima dos nossos estudantes ${ }^{10}$. Alguns foram orientados a procurar serviços de apoio da universidade, como o programa de acolhimento emocional on-line criado durante a pandemia ou o programa de apoio comunitário, com suporte da psicóloga do campus, em razão da angústia e ansiedade, sintomas frequentes nos estudantes de Medicina11,12,13.

Pelos registros, ficou muito claro que os estudantes demostraram que estavam muito atentos às políticas nacionais e com bastante engajamento social. Sentiamse sobrecarregados com algumas tarefas e estudos, mas compartilhavam ferramentas para ajudar uns aos outros. Ressaltavam o sentimento ruim de estarem longe dos colegas e "estudar medicina só olhando no computador", uma frustração comum a todos. A condição sanitária vigente comprometeu a adaptação ao método ativo de aprendizagem que, em anos anteriores, em situações de desenvolvimento das atividades acadêmicas sem restrições, já exigia um maior cuidado e frequentes intervenções. Para minimizar angústias, foram elaboradas em conjunto estratégias de organização de tempo dos estudos e das atividades de lazer. Após as semanas de avaliação, os mentores observaram, em todos os grupos, conversas mais descontraídas, voltadas ao descanso, às atividades físicas, ao dormir até tarde nos finais de semana e a leituras, filmes e séries.

No segundo semestre, observamos nos registros maior diversidade de assuntos inseridos nas diversas sessões, com discussões sobre resiliência em vários contextos, autonomia do médico na conduta terapêutica, medicina baseada em evidências, uso compassivo de medicamentos e fases dos protocolos de teste de medicamentos e vacinas. Houve ainda a menção a antigos professores da escola e às contribuições deles aos cenários médicos nacional e internacional, além reflexões sobre algumas perdas relacionadas à parceria fraterna da vida acadêmica em 2020, como festas e jogos universitários.

Constatamos também que os estudantes mostravam maturidade nas observações que faziam, considerando que os professores, coordenadores e diretores foram muito atuantes e solícitos desde o início do curso. Esse reconhecimento ficou evidente nas diversas mudanças de estratégias e adequações das atividades, inicialmente para o modo remoto e na reinserção presencial, definidas de acordo com orientações dos órgãos responsáveis. Com o retorno das atividades presenciais, todos os mentores pontuaram que houve grande entusiasmo dos estudantes quando foram conhecer as unidades básicas 
de saúde (UBS), ao frequentarem os laboratórios de anatomia e histologia, nas aulas de habilidades simuladas com atores e tiveram a oportunidade de conhecer pessoalmente alguns professores que consideraram exigentes, atenciosos e importantes sustentáculos para o seu crescimento.

O encerramento do ano ocorreu com grande alívio após grandes discussões sobre as avaliações e recuperações. Muitos estudantes mantiveram contato com seus mentores em canal privado mesmo durante o período de férias.

\section{DISCUSSÃO}

A mentoria não trata apenas do desempenho acadêmico, mas também proporciona uma visão mais ampla das questões relacionadas ao estudante e seu contexto ${ }^{2}$. Trata-se de uma experiência que pode favorecer o desenvolvimento profissional e pessoal dos estudantes envolvidos ao instigar o interesse pela pesquisa e ajudar no planejamento das trajetórias ${ }^{14,15}$. A diversidade de assuntos abordados e a liberdade para transitar entre eles protegeram e engajaram os estudantes. $\mathrm{O}$ mentor tem o papel de auxiliar os estudantes a compreender melhor o significado mais amplo dos acontecimentos que os cercam ${ }^{16}$.

Ao final das sessões, o grupo realizava uma avaliação do encontro, com frequência descrita como produtiva e proporcionando a sensação de conforto. A mentoria demonstrou ser um ambiente em que se podem discutir quaisquer assuntos ou problemas, habilidades e valores ${ }^{17} \mathrm{e}$, em situações mais individualizadas, permitiu que conversas entre estudantes e mentor fossem feitas após a sessão ou a qualquer momento na rede social privada.

A cada semana, foi possível identificar progresso nas discussões e na capacidade de argumentação, articulação e enfrentamento dos desafios propostos pelo início da complexa formação em medicina. O senso de pertencimento e participação, o entendimento de que alguns problemas são coletivos e vivenciados pelo restante do grupo auxiliaram na ampliação do modo de encarar as dificuldades e se relacionam com a inteligência emocional, fundamental para proteção dos estudantes e desenvolvimento da mentoria ${ }^{17,18}$. Refletiram até o senso de auto-humanidade descrito por Kristin Neff como um dos fatores de compaixão e autocompaixão ${ }^{19}$.

A possibilidade de compartilhar dificuldades vividas por mentores, estudantes e instituição durante um ano de pandemia foi importante na compreensão de situações complexas e difíceis ${ }^{20}$. À visão livre do cansaço, em ambiente protegido, observou-se o desenvolvimento de habilidades importantes, com pontuações mais claras e mais brandas para o olhar crítico e contestador, confirmando os benefícios da mentoria não apenas para os estudantes mentorados, mas também para os mentores e para a instituição ${ }^{21}$.

\section{CONCLUSÕES}

Nas sessões de mentoria, os estudantes e mentores cultivaram um espaço para discussão de suas rotinas no desenvolvimento do ensino e aprendizagem, permitindo correções de rumo importantes para o aprimoramento desses objetivos. Mesmo no cenário atípico vivenciado, os mentores externaram a sensação de que os estudantes saíam das reuniões mais leves, percebendo que enfrentavam problemas semelhantes e trabalhavam em conjunto na busca de soluções.

A abordagem respondendo livremente às demandas dos estudantes sem um roteiro predeterminado e a possibilidade de existir um caminho a ser seguido com alguns tópicos para guiar os encontros parecem mais adequadas que uma programação engessada, comum e formal. Roteiros prévios foram importantes em momentos em que os mentores sentiam que a reunião conduzida pela demanda não estava contribuindo ou que algum evento externo exigia uma intervenção teórica ou reflexiva para a manutenção da saúde mental do grupo.

Alguns mentores apresentaram, ao início do ano, um grau de ansiedade antes das reuniões, principalmente por não terem um roteiro a ser seguido, como em outras atividades em que atuavam como docentes e na adaptação ao modo virtual dos encontros. Mas sempre foi muito clara a descrição de agradável sensação de dever cumprido e de estar contribuindo para o bom desenvolvimento acadêmico e pessoal dos estudantes ao deixá-los à vontade para falar aberta e francamente o que precisavam e sentiam.

Nessa transição do ambiente presencial para o virtual, solução encontrada para a progressão do ano letivo, foi necessário muito empenho dos mentores, atuando como verdadeira interface entre os estudantes e a coordenação da série na superação dos desafios da inserção abrupta das novas tecnologias na prática acadêmica.

\section{AGRADECIMENTOS}

Os mentores agradecem aos alunos do primeiro ano do curso de Medicina, parceiros nesta jornada; à coordenação de eixo do primeiro ano e à diretoria do curso de Medicina, que proporcionaram à realização da mentoria suporte e adaptações necessários. Um agradecimento especial a Camila Pallazo e Antônio Pedro Maricatto competentes membros da equipe de apoio às atividades acadêmicas virtuais ou presenciais da escola.

\section{CONTRIBUIÇÃO DOS AUTORES}

Todos os autores contribuíram com a fundamentação teórica, recuperação dos relatos em grupo privado, análise do discurso, categorização do conteúdo e construção do texto. 


\section{CONFLITO DE INTERESSES}

Não houve conflito de interesses.

\section{FINANCIAMENTO}

Não houve financiamento.

\section{REFERÊNCIAS}

1. Bhatnagar V, Diaz S, Bucur PA. The need for more mentorship in medical school. Cureus. 2020 May 6;12(5):e7984.

2. Crosby, R.M. Harden J. AMEE Guide no 20: The good teacher is more than a lecturer - the twelve roles of the teacher. Med Teach. 2020 Jan 3;22(4):334-47.

3. Boucher D, Martin S, Xi AS, Rialon KL. Early career development and graduate medical education leadership pathways. J Grad Med Educ. 2020 Oct 10;12(5):644-6.

4. Cassimiro EE. Frequência do uso de psicofármacos entre jovens estudantes que cursam pré-vestibular. Adolesc e Saude. 2012;9(4):27-36.

5. Akinla $\mathrm{O}$, Hagan $\mathrm{P}$, Atiomo W. A systematic review of the literature describing the outcomes of near-peer mentoring programs for first year medical students. BMC Med Educ. 2018 Dec 8;18(1):98.

6. Santos BM, Cordeiro MEC, Schneider IJC, Ceccon RF. Educação médica durante a pandemia da Covid-19: uma revisão de escopo. Rev Bras Educ Med. 2020;44(supl 1):e0139.

7. Pontifícia Universidade Católica de São Paulo. Projeto Pedagógico do curso de Medicina da Faculdade de Ciências Médicas e da Saúde. PUCSP; 2018.

8. Pavan MV, Senger MH, Marques W. Determinantes externos e internos da reforma curricular do curso de Medicina da Pontifícia Universidade Católica de São Paulo. Rev Fac Cienc Med Sorocaba. 13 nov 2017;19(3):127-32.

9. Macedo LC, Larocca LM, Chaves MMN, Mazza V de A. Análise do discurso: uma reflexão para pesquisar em saúde. Interface Comun Saúde Educ. set 2008;12(26):649-57.
10. Ministério da Educação. Portaria no 345, de 19 de março de 2020 [acesso em 22 de fev. de 2021]. Disponível em: https://www. in.gov.br/en/web/dou/-/portaria-n-345-de-19-de-marco-de-2020248881422? inheritRedirect $=$ true\&redirect $=\% 2 \mathrm{Fweb} \% 2 \mathrm{Fguest} \% 2$ Fsearch\%3FqSearch\%3DPortaria\%2520345\%2520de\%252019\%2520de\%2520mar\%25C3\%25A7o\%2520de\%25202020.

11. Lima VV. Espiral construtivista: uma metodologia ativa de ensinoaprendizagem. Interface Comun Saúde Educ. 27 out 2016;21(61):421-34.

12. Vasconcelos TC, Dias BRT, Andrade LR, Melo GF, Barbosa L, Souza E. Prevalência de sintomas de ansiedade e depressão em estudantes de Medicina. Rev Bras Educ Med. 2015;39(1):135-42.

13. Pacheco JPG, Giacomin HT, Tam WW, Ribeiro TB, Arab C, Bezerra IM, et al Mental health problems among medical students in Brazil: a systematic review and meta-analysis. Rev Bras Psiquiatr. 2017;39:369-78.

14. Kazerooni AR, Amini M, Tabari P, Moosavi M. Peer mentoring for medical students during the COVID-19 pandemic via a social media platform. Med Educ. 2020;54(8):762-3.

15. Frei E, Stamm M, Buddeberg-Fischer B. Mentoring programs for medical students - a review of the PubMed literature 2000-2008. BMC Med Educ 2010;10(1):32-46

16. Megginson D. Mentoring in action: a practical guide (2nd ed.). Hum Resour Manag Int Dig. 2006 Dec;14(7):hrmid.2006.04414gae.002.

17. Burgess A, van Diggele C, Mellis C. Mentorship in the health professions: $a$ review. Clin Teach. 2018 June;15(3):197-202.

18. Moeller RW, Seehuus M, Peisch V. Emotional intelligence, belongingness, and mental health in college students. Front Psychol. 2020;11(93):1-10.

19. Dahm KA, Meyer EC, Neff KD, Kimbrel NA, Gulliver SB, Morissette SB Mindfulness, self-compassion, posttraumatic stress disorder symptoms, and functional disability in U.S. Iraq and Afghanistan war veterans. J Trauma Stress. 2015 Oct;28(5):460-4.

20. Rose S. Medical student education in the time of COVID-19. JAMA. 2020 June 2;323(21):2131-32.

21. Nimmons D, Giny S, Rosenthal J. Medical student mentoring programs: current insights. Adv Med Educ Pract. 2019 Mar;10:113-23. 